\title{
Solutions to Some Real-Life Problems Based on Mathematical Modeling and Functional Minimization
}

\author{
Oleksii Babaskin ${ }^{1}$ and Danilo Tadeo" \\ ${ }^{1}$ Yongsan International School of Seoul, Seoul, Republic of South Korea \\ \#Advisor
}

\section{$\underline{\text { ABSTRACT }}$}

It is useful to build mathematical models that can describe, predict, and explain real-life phenomena. This paper features the functional dependency model and the square of this functional dependency which hold significant information. A mathematical model is developed that relates these functional dependencies to the average value of the function, showing that for an arbitrary well-behaved function, the definite integral of the square of the function over a finite interval is minimal when the function is constant over the interval. In addition, the model's validity and accuracy in representing real-world problems for different situations in physics evaluated.

\section{Introduction}

During a discussion I had with my father, he brought up an interesting phenomenon in antenna theory: the more homogeneously the current is distributed over the antenna, the higher its performance is, provided all the other quantities (matching condition, total current, size, and material conductivity) are equal.

The operation of the radio communications systems depends on antenna performance. This is described by antenna theory. Antenna theory explains how to maximize the antenna's capability of transmitting and receiving electromagnetic (EM) waves. An antenna is a structure that receives and transmits radio EM waves. To achieve a maximum power transfer or optimal electric power into EM radiation conversion, the reflections between the antenna and the transceiver should be minimized by impedance matching of the two devices. Perfect impedance matching assumes that complex impedance of the antenna and the source are complex conjugates of each other to minimize the return loss (Balanis, 2005). If these conditions are satisfied - ensuring all the other factors are constant and minimizing the Ohmic losses in the antenna - then maximizing of the antenna performance can be achieved. Ohmic loss particularly depends on electric current density distribution over the antenna. As mentioned, and to be proven in this paper, increasing current distribution homogeneity reduces the Ohmic loss and would lead to a higher antenna performance due to higher energy conversion efficiency. The discussion I had with my father made me think about the energy-like quantities in systems and optimization problems concerning them.

Mathematical models are used to explain, solve, and predict real-life situations (Rangel, et al., 2016). The first step in developing a mathematical model is to recognize the problem, followed by expressing the problem mathematically. When building a mathematical model, it is crucial to identify and relate the variables in the problem. The next step is to set up a framework of the model that can be an equation or a set of equations. A strategy to solve the equations is then developed and the results are interpreted and linked to real-life situations.

Considering a variety of variables that can be present in a phenomenon, it is advisable to introduce a functional dependency (FD) between these variables. The definite integral of a quantity involving functionals is fundamentally important to understand different physical phenomena (Susskind \& Friedman, 2014). One then has to do an analysis that might need specific techniques in calculus to make some useful predictions (Bayin, 2019).

The great mathematician Euler (Euler, 1744) stated that "all of nature behaves according to some principle of a maximum or a minimum". Extremal problems are very important in the application of mathematics to real-world 
problems (Kristály et al 2013). Calculus of variations is a branch of mathematics concerned with the problem of finding a function for which the value of a certain integral is either maximum or minimum (Bliss, 1947; Hadley, 1964). This particular branch of mathematics is used in various formulations of the principles of D'Alembert, Lagrange in classical physics, the Fermat principle in electrodynamics, quantum physics, geodesics problems, and stochastic calculus (Calder, 2020).

The principle of least action formulated by Euler and Maupertuis was originally applicable only to conservative systems. Later it was further developed and adapted to nonconservative systems including systems with velocitydependent forces (Gray, 2004).

In this paper we have applied the principle of least action in the presence of velocity-dependent forces and investigated the possibility of using the variational principle to different real-life situations. We considered a special case of functionals where the integrand depends only upon a function or the square of a one-independent-variable function. A mathematical model was developed and the concept of "energy minimization" was applied to analyze these situations. We suggested a simple mathematical approach to search for the minimum function of the squared FD, given the mean value of the FD constant. Such a setup is a special case of a generalized isoperimetric problem (Pontryagin, et al., 1962). Isoperimetric problems are common in science and engineering applications, where it's often desirable to maximize or minimize a physical variable through shape variation under certain constraints (Curtis, 2004). Finally, we showed that applying the mathematical model and the calculus of variation to different situations is simple albeit, general enough to sufficiently describe these phenomena.

\section{Mathematical Model Development}

In this section we will develop the mathematical model adopted in our study. Consider that $y=f(x)$ is a function defined over the interval $[a, b]$. The average value of the function $f(x)$ over the interval $[a, b]$ is noted $\bar{f}$ and is defined as:

$\int_{a}^{b} f(x) d x \equiv \bar{f}(b-a)=c$

Equation 1

Figure 1(i) shows in black the graph of a function $y=f(x)$ over the interval $[a, b]$ and in red a visual representation of the average value calculated using Equation 1.

Now let us consider the square of the function $f(x)$, i.e., $f^{2}(x)$. The definite integral of the function $f^{2}(x)$ over the interval $[a, b]$ is defined as $\int_{a}^{b} f^{2}(x) d x$. Figure 1(ii) shows the graph of the function $f^{2}(x)$ over the interval $[a, b]$. The dashed area under the curve represents $\int_{a}^{b} f^{2}(x) d x$. The upper red line represents a visual representation of $\bar{f}^{2}$. 


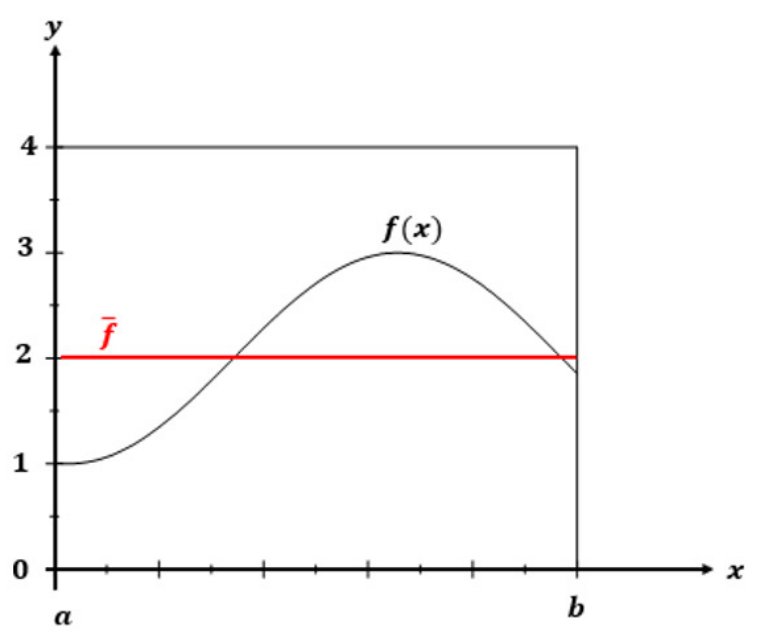

(i)

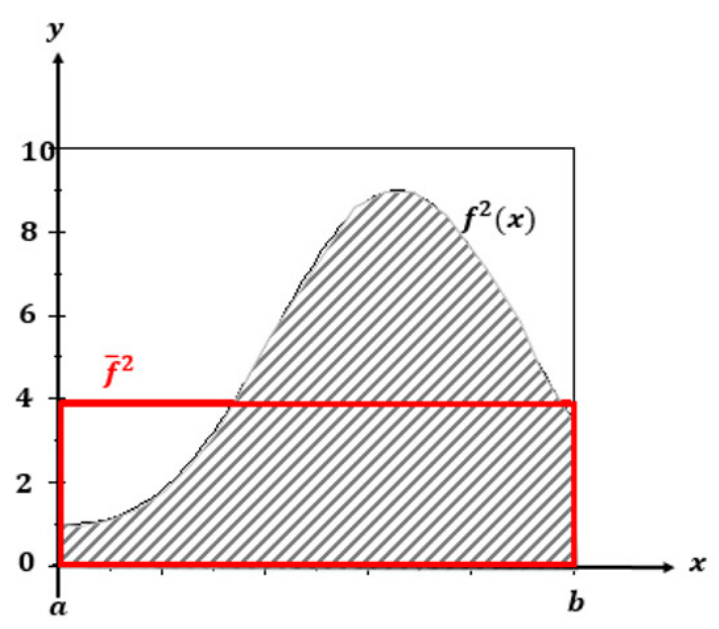

(ii)

Figure 1. (i) In black we have graphical representation of a function $f(x)$ over the interval [a,b] and in red the average value of the function $\bar{f}$ is calculated using Equation 1. (ii) In black we have graphical representation of the function $f^{2}(x)$ over the interval [a,b]. The dashed area under the curve represents $\int_{a}^{b} f^{2}(x) d x$. The area of the red rectangle represents $\int_{a}^{b} \bar{f}^{2} d x$.

We aim to demonstrate that under the constraint of the form of the Equation 1 the value of the integral $\int_{a}^{b} f^{2}(x) d x$ is minimal, when $f(x)$ is constant over the interval $[a, b]$ (and, therefore, is equal to $\bar{f}$ ). In other words, it requires that the function $f(x)$ does not vary from the interval $[a, b]$.

In terms of calculus of variations, the basic problem is to find a function $f(x)$ for which $\int_{a}^{b} f^{2}(x) d x$ is minimal. To do that, we will represent the function $y=f(x)$ as the sum of the average value $\bar{f}$ and a variation parameter $\delta(x)$.

$y=f(x)=\bar{f}+\delta(x)$

Equation 2

We will consider by definition that the average value of $\overline{\delta(x)}=\int_{a}^{b} \delta(x) d x=0$ over the interval $[a, b]$. We can obtain:

$\int_{a}^{b} f^{2}(x) d x=\int_{a}^{b}(\bar{f}+\delta(x))^{2} d x$

$\int_{a}^{b} f^{2}(x) d x=\bar{f}^{2} \int_{a}^{b} d x+\int_{a}^{b} \delta(x)^{2} d x+2 \bar{f} \int_{a}^{b} \delta(x) d x$

$\int_{a}^{b} f^{2}(x) d x=\bar{f}^{2}(b-a)+\int_{a}^{b} \delta(x)^{2} d x$

Equation 3

It is obvious that $\int_{a}^{b} f^{2}(x) d x$ is minimal, when $\delta(x) \equiv 0$ i.e., $f(x)=\bar{f}$. In this case the value of $\int_{a}^{b} f^{2}(x) d x$ is equal to $\bar{f}^{2}(b-a)$ that represents the area of the red rectangle shown in figure 1 (ii). Therefore, the definite integral of the square of the function $\int_{a}^{b} f^{2}(x) d x$ over a finite interval $[a, b]$ is minimal when the function is constant over the interval.

\section{Results and Applications}

In this section, the mathematical model developed is applied to describe different real-life situations.

\section{Minimizing energy consumption in the presence of viscous force}


Mechanical energy can be defined as the ability to move an object over some distance. If no frictional forces are acting on the object, the total mechanical energy is conserved. When the object moves through fluids, the mechanical kinetic energy is irreversibly converted to thermal energy due to frictional forces induced by fluid viscosity.

Let's consider the case of an object moving in a viscous medium and look for the conditions to minimize the mechanical energy consumption during its movement. The total displacement of an object moving in a viscous medium along the $\mathrm{x}$-axis during the time interval $[\mathrm{a}, \mathrm{b}]$ is the constraint in this problem and it is defined as:

$d=\int_{a}^{b} v(t) d t$

Equation 4

Where $v(t)$ is the velocity-time function. The average velocity $\bar{v}$ over the time interval $[a, b]$ would be:

$\bar{v}=\frac{1}{(b-a)} \int_{a}^{b} v(t) d t=\frac{d}{(b-a)}$

Equation 5

The work done by the force of viscous friction represents the energy lost by the object during the time interval $[a, b]$. The force of viscous friction is defined as:

$F_{\text {viscous }}=\eta v(t)$

Equation 6

Where $\eta$ is the coefficient of viscosity. We can express the work done by $F_{\text {viscous }}$ on the system of the particle as it moves from $x_{i}$ to $x_{f}$ as:

$W_{\text {viscous }}=\int_{x_{i}}^{x_{f}} F_{v i s c o u s} d x=\int_{x_{i}}^{x_{f}} \eta v(t) d x=\int_{x_{i}}^{x_{f}} \eta v(t) \frac{d x}{d t} d t=\eta \int_{a}^{b} v^{2}(t) d t$

Equation 7

We consider $E_{\text {fuel }}$ the energy needed to compensate for friction losses. Thus, the fuel energy consumed over the time interval $[\mathrm{a}, \mathrm{b}]$ is given by:

$E_{\text {fuel }}=W_{\text {friction }}=\eta \int_{a}^{b} v(t)^{2} d t$

Equation 8

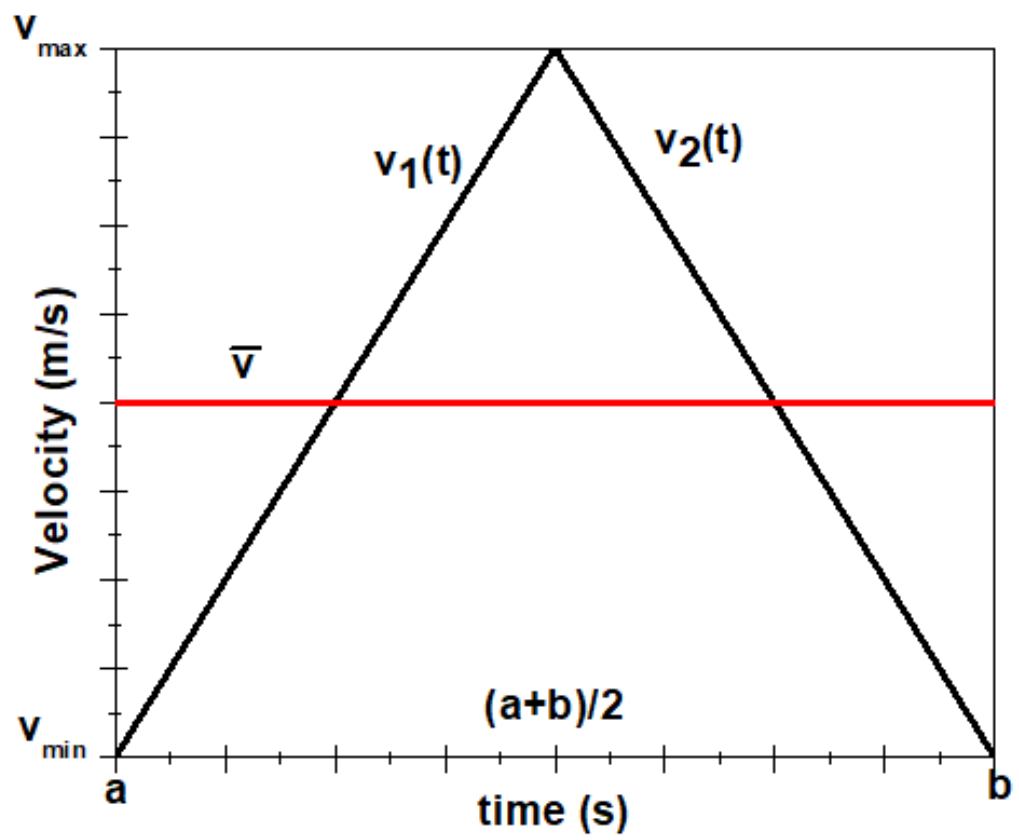

Figure 2: In black we have graphical representation of a velocity function $v(t)$ over the interval [a,b] and in red the average value of the function $\bar{v}$ calculated using Equation 1

Let us consider the case of a vehicle moving with the constant velocity $\bar{v}$ as depicted in Figure 2 (the red line) in the presence of frictional forces. The fuel energy needed to compensate for the energy losses due to frictional forces is equal to $\eta \bar{v}^{2}(b-a)$. 
Then, we assume that the vehicle is moving with a non-constant velocity $v(t)$ over the time interval $[a, b]$. We have considered a function $v(t)$ having an average value equal to $\bar{v}$ over the time interval $[a, b]$. The velocity of the vehicle increases gradually to reach its maximum value $v_{\max }$ at $t=(a+b) / 2$, and then decreases gradually to reach its minimum value $v_{\min }$ at $t=b$ as indicated in figure 2 . To model the acceleration/deceleration of the vehicle due to in the time interval $[a, b]$, we make use of the following set of equations of $v(t)$

$v(t)=\left\{\begin{array}{cl}v_{1}(t)=2\left(\frac{v_{\max }-v_{\min }}{b-a}\right) t+v_{\min } & \text { for } a<t<\frac{a+b}{2} \\ v_{2}(t)=-2\left(\frac{v_{\max }-v_{\min }}{b-a}\right)\left(t-\frac{a+b}{2}\right)+v_{\max } & \text { for } \frac{a+b}{2}<t<b\end{array}\right.$

Equation 9

For the following specific case $\left(v_{\min }=0, v_{\max }=4 \mathrm{~m} / \mathrm{s}, a=0 \mathrm{~s}, b=80 \mathrm{~s}\right)$ the fuel energy consumption is equal to $1.15 \eta \bar{v}^{2}(b-a)$. Thus, to compensate the energy losses due to frictional forces in the case of non-constant velocity our model predicts an additional $15 \%$ fuel energy consumption compared to the case where the vehicle was moving with a constant velocity $\bar{v}$.

In this section we used a simple mathematical model that can predict the energy consumption of an object (for instance a vehicle) moving in the presence of a friction force. We have concluded that to minimize the energy consumption, the velocity of the object should stay constant. Our result is in accordance with an early study (Barth et al. 2008) on the energy consumption due to traffic congestion. It was shown that vehicle fuel consumption near signalized intersections is considerably enhanced by the vehicle slowdown and acceleration actions. Additionally, Barth et al observed that even minor variations in vehicle's speed can have a big impact on the energy consumption (Barth et al 2008). For example, reducing the number and severity of individual accelerations and decelerations may decrease energy consumption up to $10 \%$. Different strategies are adapted to maintain the vehicle speed constant. One of them is the intelligent speed adaptation (ISA) which has the ability to eliminate the number of stops leading to speed variation during congestion time. Hence the reduction of fuel consumption, due to stabilization of the vehicle speed, can minimize the pollutant emission and preserve the environment clean and green. Developing mathematical modeling for accurate estimation of the fuel consumption was and continues to be crucial in the development of transportation systems. Finally, the proposed model, in this section, has a simple and flexible shape that allows it to be amelioratedin the future by incorporating additional parameters such as road slope.

\section{Minimizing Ohmic loss in a conductor wire}

A current $I$ is flowing along a conducting ribbon, where a line transversal to the current's flow is the X-axis. The total current $I$, flowing through the conductor between $x_{i}=a$ and $x_{f}=b$ is the constraint in this problem and it would be:

$I=\int_{a}^{b} J(x) d x$

Equation 10

$J(x)$ is the linear current density expressing the current per unit width $(\mathrm{A} / \mathrm{m})$

Then, the average current density over the interval $[a, b]$ :

$\bar{J}=\frac{1}{(b-a)} \int_{a}^{b} J(x) d x$

Equation 11

Resistance in conducting wires produces a loss of energy. Energy from electric field is converted into heat. The power dissipation (Ohmic loss per unit width) when the current flows between $x_{i}=a$ and $x_{f}=b$ would be: $P_{\text {heat }}=\rho \int_{a}^{b} J^{2}(x) d x$

Equation 12

Where $\rho$ is the resistivity. In order to minimize Ohmic loss into the conductor wire, the value of the $\int_{a}^{b} J^{2}(x) d x$ should be minimized. Based on our mathematical model, we can assert that the linear current density along the conductor wire must be homogenous (and equal to its average value). 


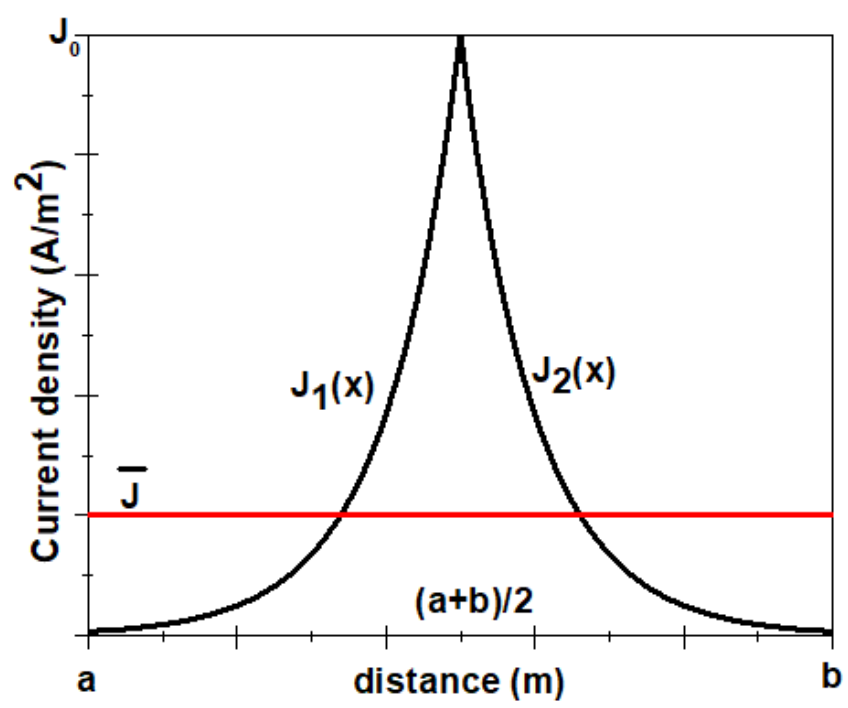

Figure 3: In black we have graphical representation of a current density function $J(x)$ over the interval $[a, b]$ and in red the average value of the function $\bar{J}$ calculated using Equation 1

Let us consider a conductive ribbon that carries a uniform current density $\bar{J}$ over an interval $[a, b]$. The ohmic losses over the interval $[a, b]$ are equal to $\rho J^{2}(b-a)$. Then, we consider the case where the current density $J(x)$ is maximum at the center of the ribbon and drops exponentially from the center in a way that can be quantified in terms of a parameter $\delta$. The current density variation represented in figure 3 is modeled using the following set of equations

$J(x)= \begin{cases}J_{0} e^{-\left[\frac{a+b}{2}-x\right] / \delta} & \text { for } a<x<\frac{a+b}{2} \\ J_{0} e^{\left[\frac{a+b}{2}-x\right] / \delta} & \text { for } \frac{a+b}{2}<x<b\end{cases}$

Equation 13

We have considered a function $J(x)$ having an average value equal to $\bar{J}$ over the time interval $[a, b]$. For the following specific case $(a=0, b=8 \mathrm{~mm}$ and $\delta=0.66 \mathrm{~mm}$ ) ohmic losses are increased by $20 \%$ compared to constant current density case.

In order to achieve the highest performance of an electric circuit, it is important to minimize the ohmic losses in the conductor. In the case of alternative current, the current density distribution become inhomogeneous $J(x)$ variable) inside the conductor due to the alternating magnetic field and "eddy currents". Thus, to minimize additional ohmic losses, conductor designs with very low induced "eddy current" become more and more important. Milliken type conductor and Litz wire are two conductors specifically designed to reduce ohmic losses. Milliken conductor is divided generally into six insulated segments around a hollow central channel. Litz wire is a special type of wire consisting of many thin wires; individually coated with an insulating film and twisted together. These two designs lead to a homogenization (constant) of the current density (Willen et al, 2008) inside each segment and thus to the reduction of ohmic losses. This result is in accordance with what was predicted in equation 11 reinforcing the robustness of our model.

\section{Conclusion}

In this paper, we have developed a simple mathematical model to analyze and predict real-life situations. The basic idea behind this model is to minimize the value of the definite integral of the square of a function describing a system under the constraint for average value of this function. We have found that to minimize the value of the definite integral over an interval $[a, b]$ the function should be constant over this interval. Then we have applied this model to 
some real-life systems in physics. Finally, this simple model can be further developed and refined to be applied to other situations and more complex systems.

\section{Acknowledgments}

I would like to thank my advisor Danilo Jr Tadeo and my father Alexandr Babaskin for helping me with this project

\section{References}

Balanis, C. A., 2005. Antenna theory: Analysis and design. 3rd edition ed. Wiley. Bayın, S., 2019. Essentials of Mathematical Methods in Science and Engineering. Wiley. Barth \& Boriboonsomsin, 2008; Real-World Carbon Dioxide Impacts of Traffic Congestion, Journal of the Transportation Research Board, No. 2058, Transportation Research Board of the National Academies, Washington, D.C., 2008, pp. 163- 171 .

Bliss, G., 1947. Lectures on the calculus of variations, Chicago Univ. Press. Calder, J., 2020. The Calculus of Variations, University of Minnesota.

Curtis, J., (2004) Complementary Extremum Principles for Isoperimetric Optimization Problems.

Hadley, G., 1964. Nonlinear and dynamic programming. Addison-Wesley.

Gray, C.G., G. Karl and V.A. Novikov (2004). "Progress in Classical and Quantum Variational Principles", Rep. Prog. Phys. 67, 159-208.

Kristály, A. Rădulescu, V. Vagra, C. 2013 Variational Principles in Mathematical Physics, Geometry, and Economics. Cambridge University Press: 05 June 2013.

Leonhard Euler, (1744) Methodus Inveniendi Lineas Curvas Maximi Minive Proprietate Gaudentes. Bousquet, Lausanne \& Geneva. 320 pages. Reprinted in Leonhardi Euleri Opera Omnia: Series I vol 24. (1952) C. Cartheodory (ed.) Orell Fuessli, Zurich. scanned copy of complete text at The Euler Archive, Dartmouth.

Pontryagin, L., Boltayanskii, V., Gamkrelidze, R. \& Mishchenko, E., 1962. The mathematical theory of optimal processes, Wiley.

Rangel, R., Magaña, M. \& Azpeitia, R., 2016. Mathematical Modeling in Problem Situations of Daily Life. Journal of Education and Human Development, Volume 5, pp. 62-76.

Susskind, L. \& Friedman, A., 2014. Quantum mechanics: The Theoretical Minimum: What you need to know to start doing physics, Basic books.

Willen, D \& Waschk, V, 2008, Electrical sector cable of the Milliken-type, EP2141709A2 European Patent Office. 